Article

\title{
The Lipid Paradox Among Acute Ischemic Stroke Patients-A Retrospective Study of Outcomes and Complications
}

\author{
Urvish Patel $^{1, *(\mathbb{D})}$, Preeti Malik ${ }^{2}$, Mihir Dave ${ }^{3}$, Matthew S. DeMasi ${ }^{4}$, Abhishek Lunagariya ${ }^{1}$, \\ Vishal B. Jani ${ }^{1,+}$ and Mandip S. Dhamoon ${ }^{5,+}$ \\ Department of Neurology, Creighton University School of Medicine, Omaha, NE 68124, USA \\ Department of Public Health, Icahn School of Medicine at Mount Sinai, New York, NY 10029, USA \\ Department of Internal Medicine, University of Nevada Reno School of Medicine, Reno, NV 89102, USA \\ Department of Internal Medicine, Albert Einstein College of Medicine, Bronx, NY 10461, USA \\ Department of Neurology, Icahn School of Medicine at Mount Sinai, New York, NY 10029, USA \\ * Correspondence: dr.urvish.patel@gmail.com; Tel: +1-(201)-936-6715 \\ + Both authors contributed equally.
}

Received: 24 June 2019; Accepted: 13 August 2019; Published: 13 August 2019

\begin{abstract}
Background and objectives: The Studies have suggested hypercholesterolemia is a risk factor for cerebrovascular disease. However, few of the studies with a small number of patients had tested the effect of hypercholesterolemia on the outcomes and complications among acute ischemic stroke (AIS) patients. We hypothesized that lipid disorders (LDs), though risk factors for AIS, were associated with better outcomes and fewer post-stroke complications. Materials and Method: We performed a retrospective analysis of the Nationwide Inpatient Sample (years 2003-2014) in adult hospitalizations for AIS to determine the outcomes and complications associated with LDs, using ICD-9-CM codes. In 2014, we also aimed to estimate adjusted odds of AIS in patients with LDs compared to patients without LDs. The multivariable survey logistic regression models, weighted to account for sampling strategy, were fitted to evaluate relationship of LDs with AIS among 2014 hospitalizations, and outcomes and complications amongst AIS patients from 2003-2014. Results and Conclusions: In 2014, there were 28,212,820 (2.02\% AIS and 5.50\% LDs) hospitalizations. LDs patients had higher prevalence and odds of having AIS compared with non-LDs. Between 2003-2014, of the total 4,224,924 AIS hospitalizations, 451,645 (10.69\%) had LDs. Patients with LDs had lower percentages and odds of mortality, risk of death, major/extreme disability, discharge to nursing facility, and complications including epilepsy, stroke-associated pneumonia, GI-bleeding and hemorrhagic-transformation compared to non-LDs. Although LDs are risk factors for AIS, concurrent LDs in AIS is not only associated with lower mortality and disability but also lower post-stroke complications and higher chance of discharge to home.
\end{abstract}

Keywords: stroke; hyperlipidemia; epilepsy; stroke associated pneumonia; gastro-intestinal hemorrhage; hemorrhagic transformation; nationwide inpatient sample; outcomes

\section{Introduction}

Hypercholesterolemia is a well-documented risk factor for cardiovascular morbidity and mortality [1-4]. However, the relationship between ischemic stroke and cholesterol is complex and appears to contain several paradoxes [5-12]. Many large-scale studies on stroke and cholesterol have not differentiated between ischemic and hemorrhagic stroke, nor among various subtypes of ischemic stroke [6,8-10]. It has been demonstrated that cholesterol may increase the risk of only certain types of stroke [13-15], and low cholesterol levels predispose to hemorrhagic stroke [10,16], thus weakening an 
association between cholesterol and all stroke. Regardless of increased cardiovascular disease risk with high cholesterol levels and decreased stroke risk with statin use, a higher cholesterol value has been associated with a better stroke outcome in several studies [17-20]. Further research has shown "reverse epidemiology" between cholesterol levels at admission, statin treatment and stroke morbidity. Earlier studies have shown a positive association between elevated admission cholesterol at ischemic stroke onset and improved short-term functional outcome [21] and 10-year survival [22].

These paradoxical observations may be related to differential associations with different stroke subtypes. Several recent studies reported that cholesterol was lowest in cardioembolic strokes $[15,23,24]$. The largest prospective cohort of 4128 adults aged $>70$ years reported that high or normal/borderline total cholesterol values were associated with good survival compared to low values [25].

We aimed to estimate odds of having AIS with LDs, and whether or not LDs in AIS patients were associated with better outcomes.

\section{Materials and Methods}

Data was obtained from the Nationwide Inpatient Sample (NIS) between January 2003 and December 2014. The NIS is the largest publicly available all-payer inpatient care database in the United States and contains discharge-level data provided by states that participate in the HCUP (including a total of 46 in 2011). This administrative dataset contains data on approximately 8 million hospitalizations in 1000 hospitals that were chosen to approximate a $20 \%$ stratified sample of all US community hospitals, representing more than $95 \%$ of the national population. Criteria used for stratified sampling of hospitals into the NIS include hospital ownership, patient volume, teaching status, urban or rural location, and geographic region. Discharge weights are provided for each patient discharge record, which allow extrapolation to obtain national estimates. Each hospitalization is treated as an individual entry in the database and is coded with one principal diagnosis, up to 24 secondary diagnoses, and 15 procedural diagnoses associated with that stay. Detailed information on NIS is available at http://www.hcup-us.ahrq.gov/db/nation/nis/nisdde.jsp.

The data were taken from the Nationwide Inpatient Sample, which is a deidentified database from "Health Care Utilization Project (HCUP)" sponsored by the Agency for Healthcare Research and Quality, USA, thus informed consent or IRB approval was not needed for the study. The relevant ethical oversight and HCUP Data Use Agreement (HCUP-4Q28K90CU) were obtained for the study.

\subsection{Study Population}

We used the ninth revision of the International Classification of Diseases, clinical modification codes (ICD-9-CM) to identify adult patients admitted with a primary diagnosis of AIS (ICD-9-CM codes 433.01, 433.11, 433.21, 433.31, 433.81, 433.91, 434.01, 434.11, 434.91). These codes have been previously validated and are $35 \%$ sensitive, $99 \%$ specific, $96 \%$ positive predictive value (PPVs), and 79\% negative predictive value for the diagnosis of ischemic stroke [26]. Similarly, patients with secondary diagnosis of LDs were identified using ICD-9-CM codes 272.0, 272.1, and 272.2 (sensitivity of 27.0\%, specificity of $76.7 \%$, PPV of $71.1 \%$, and NPV of 33.1\%) [27]. We used ICD-9-CM codes to identify independent predictors (covariates), including the comorbidities of hypertension, diabetes mellitus, hypercholesterolemia, atrial fibrillation, use of anticoagulant and antiplatelet medications, chronic use of NSAIDs and aspirin, smoking (current/past), use of IV tPA, drug abuse, alcohol dependence, smoking status, mechanical thrombectomy, AV malformation, amyloidosis, atrial fibrillation, nasogastric tube, gastrostomy, endotracheal intubation, non-invasive mechanical ventilation, invasive mechanical ventilation and H. pylori infection. Supplementary Table S1 lists all ICD-9-CM codes that were used for this study.

AIS patients were stratified by LDs status. Age $<18$ years and admissions with missing data for age, sex, and race were excluded. 


\subsection{Patient and Hospital Characteristics}

Patient characteristics of interest were sex, age, race, insurance status and concomitant diagnoses as defined above. Race was defined by White (referent), African American, Hispanic, Asian or Pacific Islander, and Native American. Insurance status was defined by Medicare (referent), Medicaid, private insurance, and other/self-pay/no charge. We defined the severity of co-morbid conditions using Deyo's modification of the Charlson co-morbidity index (CCI) (Supplementary Table S2). Thirty-one facilities were considered to be teaching hospitals if they have an American Medical Association-approved residency program, are a member of the Council of Teaching Hospitals, or have a full-time equivalent interns and residents to patient's ratio of $\geq 0.25$. HCUP NIS contains data on total charges for each hospital in the databases, which represents the amount that hospitals billed for services.

\subsection{Outcomes}

We tested for associations between LDs and AIS amongst the January 2014-December 2014 dataset. We also examined outcomes such as all-cause in hospital mortality, All Patient Refined Diagnosis Related Groups (APR-DRG) risk of death (RoD), APR-DRG loss of function (LoF), discharge disposition (DD) [home vs. transfer to short-term hospital (STH), skilled nursing facility (SNF), intermediate care facility (ICF)], post-stroke complications like epilepsy, stroke-associated pneumonia (SAP), hemorrhagic transformation (HT), upper gastro-intestinal bleeding (UGIB), length of stay (LoS), and cost of hospitalization with LDs amongst AIS hospitalizations (years 2003-2014). The comparison of disability/loss of function was investigated by APR-DRGs severity between patients with LDs and patients without LDs on discharge. Similarly, risk of death was assigned using APR-DRG likelihood of death (risk of death) on discharge. APR-DRGs were assigned using software developed by 3M Health Information Systems, where score 1 indicates minor, 2-moderate, 3-major, 4-extreme loss of function or likelihood of death on discharge.

\subsection{Statistical Analysis}

All statistical analyses were performed using the weighted survey methods in SAS (version 9.4). Weighted values of patient-level observations were generated to produce a nationally representative estimate of the entire US population of hospitalized patients. Univariate analysis of differences between categorical variables was tested using the chi-square test and analysis of differences between continuous variables (LoS and cost of hospitalization) was tested using Student's t-test. Mixed-effects survey logistic regression models with weighted analysis were used for the categorical dependent variables, including LDs and outcomes of interest, in order to estimate odds ratio (OR) and 95\% confidence intervals for the association between AIS and LDs in the 2014 cohort as well as LDs and outcomes amongst AIS hospitalizations during years 2003-2014.

The hierarchical models (demographics and patient-level factors nested within hospital-level factors) were created as random effects within the model for the outcomes. In the multivariate models, we had included demographics (age, gender, race), patient-level hospitalization variables (admission day, primary payer, admission type, median household income category), hospital-level variables (hospital region, teaching versus nonteaching hospital, hospital bed size), comorbidities/concurrent conditions like hypertension, diabetes mellitus, hypercholesterolemia, atrial fibrillation, obesity, hemorrhagic transformation, smoking status, drug abuse, alcohol abuse, medication use (anticoagulant and antiplatelet medication, chronic use of aspirin), and use of IV tPA during the same hospitalization or in a different institution within the $24 \mathrm{~h}$ prior to admission to the facility, mechanical thrombectomy, gastrostomy, nasogastric tube insertion, invasive-noninvasive mechanical ventilation, and CCI. The confounders of the models were tailored according to the need of the individual model.

We investigated the link between LDs and AIS, LDs and post-AIS outcomes, and LDs and post-AIS complications by creating separate mix effect survey logistic regression models with weights to account for sampling strategy to find out:

(1) Relationship of LDs with AIS amongst year 2014 hospitalizations; 
(2) Relationship of LDs with post-AIS outcomes and complications amongst AIS hospitalizations from year 2003-2014;

Model 1: All cause in hospital mortality;

Model 2: Discharge disposition (home vs. non-home);

Model 3: APR-DRG loss of function (major/severe vs. minor/moderate);

Model 4: APR-DRG risk of death (major/severe likelihood vs. minor/moderate likelihood);

Model 5: Post-AIS early epilepsy;

Model 6: Stroke Associated Pneumonia (SAP);

Model 7: Upper gastro-intestinal bleeding (UGIB);

Model 8: Hemorrhagic transformation (HT).

For each model, C-index was calculated. All statistical tests used were 2-sided, and $p<0.05$ was deemed statistically significant.

\section{Results}

\subsection{LDs and AIS Amongst Year-2014 Hospitalizations}

We identified 28,212,820 total hospitalizations in 2014, (Figure 1A) out of which $(569,215) 2.02 \%$ and $(1,550,956) 5.50 \%$ patients were hospitalized with primary or secondary diagnosis of AIS and LDs respectively. Out of 569,215 patients with AIS, 50,005 (8.78\%) had LDs. Patients with LDs had higher prevalence of having AIS $(3.22 \%$ vs. $1.95 \%$; $p<0.0001)$ compared with non-LDs (Table 1$)$.

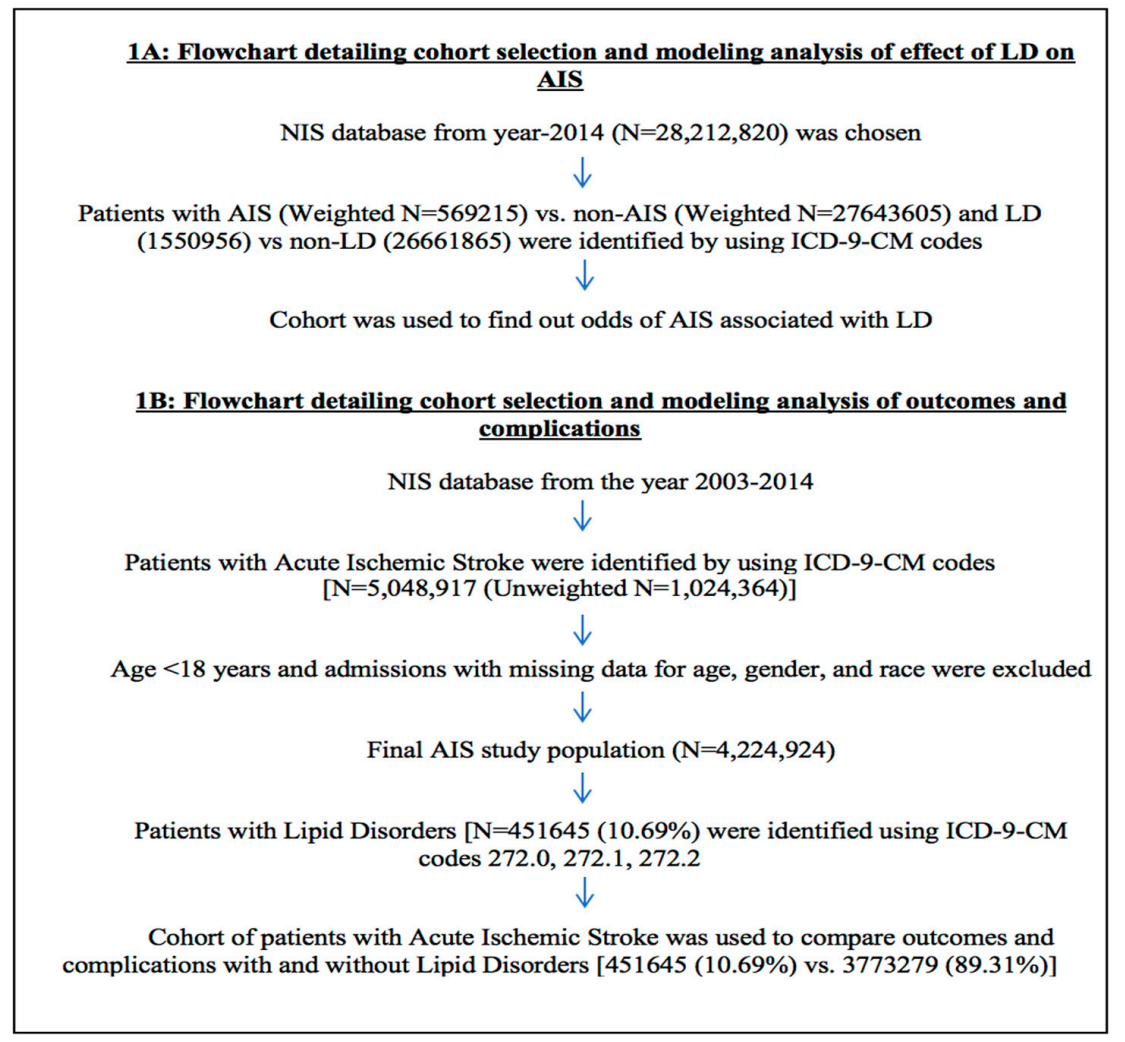

Figure 1. Flowchart detailing cohort selection and analysis modeling.

Table 1. Univariate associations of acute ischemic stroke (AIS) with lipid disorders (LDs) in the year 2014.

\begin{tabular}{ccccc}
\hline & LD & No LD & Total & $p$ Value \\
\hline AIS & $50,005(3.22 \%)$ & $519,210(1.95 \%)$ & 569,215 & \\
No AIS & $1,500,951(96.78 \%)$ & $26,142,654(98.05 \%)$ & $27,643,605$ & $<0.0001$ \\
& $1,550,956$ & $26,661,864$ & $28,212,820$ & \\
\hline
\end{tabular}


Table 2 includes regression models among 2014 hospitalizations with odds of having AIS amongst LDs vs. non-LDs after adjusting for patients' demographics, patients and hospital level characteristics, co-morbidities, and CCI. In multivariate survey logistic regression analysis, LD was associated with higher adjusted odds of having AIS (aOR: 1.18; 95\% CI:1.15-1.20; $p<0.0001$ ) compared to non-LDs. The AUC or C statistic of the ROC was used to validate the accuracy of the regressions. The AUC was 0.88 , which indicates a precise/good model.

Table 2. Multivariate logistic regression analysis of predictors of AIS and associations with LDs among year 2014 hospitalizations.

\begin{tabular}{|c|c|c|c|c|}
\hline & \multirow[t]{2}{*}{ OR } & \multicolumn{2}{|c|}{ 95\% Confidence Limits } & \multirow[t]{2}{*}{$p$ Value } \\
\hline & & LL & UL & \\
\hline No Lipid Disorders & \multicolumn{4}{|c|}{ Reference } \\
\hline Lipid Disorders & 1.18 & 1.15 & 1.20 & $<0.0001$ \\
\hline \multicolumn{5}{|c|}{ Demographics of Patients } \\
\hline Age (Years) & 1.02 & 1.02 & 1.02 & $<0.0001$ \\
\hline \multicolumn{5}{|l|}{ Gender } \\
\hline Female & \multicolumn{4}{|c|}{ Reference } \\
\hline Male & 1.00 & 0.99 & 1.01 & 0.8272 \\
\hline \multicolumn{5}{|l|}{ Race } \\
\hline White & \multicolumn{4}{|c|}{ Reference } \\
\hline African American & 1.17 & 1.15 & 1.19 & $<0.0001$ \\
\hline Hispanic & 0.90 & 0.88 & 0.93 & $<0.0001$ \\
\hline Asian or Pacific Islander & 1.12 & 1.07 & 1.16 & $<0.0001$ \\
\hline Native American & 0.92 & 0.84 & 1.01 & 0.0793 \\
\hline \multicolumn{5}{|c|}{ Characteristics of Patients } \\
\hline \multicolumn{5}{|c|}{$\begin{array}{l}\text { Median Household Income } \\
\text { Category for patient's Zip code * }\end{array}$} \\
\hline $0-25$ th percentile & \multicolumn{4}{|c|}{ Reference } \\
\hline 26-50th percentile & 1.03 & 1.01 & 1.04 & 0.0038 \\
\hline $51-75$ th percentile & 1.02 & 1.01 & 1.04 & 0.0127 \\
\hline 76-100th percentile & 1.01 & 0.99 & 1.03 & 0.2592 \\
\hline \multicolumn{5}{|l|}{ Primary Payer } \\
\hline Medicare & \multicolumn{4}{|c|}{ Reference } \\
\hline Medicaid & 1.04 & 1.01 & 1.07 & 0.0025 \\
\hline Private Insurance & 1.30 & 1.27 & 1.32 & $<0.0001$ \\
\hline Other/Self-pay/No charge & 1.46 & 1.42 & 1.51 & $<0.0001$ \\
\hline \multicolumn{5}{|l|}{ Admission type } \\
\hline Non-elective & \multicolumn{4}{|c|}{ Reference } \\
\hline Elective & 0.29 & 0.28 & 0.30 & $<0.0001$ \\
\hline \multicolumn{5}{|l|}{ Admission day } \\
\hline Weekday & \multicolumn{4}{|c|}{ Reference } \\
\hline Weekend & 1.13 & 1.11 & 1.15 & $<0.0001$ \\
\hline
\end{tabular}


Table 2. Cont.

\begin{tabular}{|c|c|c|c|c|}
\hline & \multirow[t]{2}{*}{ OR } & \multicolumn{2}{|c|}{ 95\% Confidence Limits } & \multirow[t]{2}{*}{$p$ Value } \\
\hline & & LL & UL & \\
\hline \multicolumn{5}{|c|}{ Characteristics of Hospitals } \\
\hline \multicolumn{5}{|l|}{ Bed-size of hospital $^{\dagger}$} \\
\hline Small & \multicolumn{4}{|c|}{ Reference } \\
\hline Medium & 1.11 & 1.09 & 1.13 & $<0.0001$ \\
\hline Large & 1.15 & 1.13 & 1.17 & $<0.0001$ \\
\hline \multicolumn{5}{|l|}{ Hospital Location \& Teaching Status } \\
\hline Rural & \multicolumn{4}{|c|}{ Reference } \\
\hline Urban Non-teaching & 1.04 & 1.02 & 1.07 & 0.0019 \\
\hline Urban Teaching & 1.14 & 1.12 & 1.17 & $<0.0001$ \\
\hline \multicolumn{5}{|l|}{ Hospital Region } \\
\hline Northeast & \multicolumn{4}{|c|}{ Reference } \\
\hline Midwest & 1.14 & 1.12 & 1.17 & $<0.0001$ \\
\hline South & 1.23 & 1.21 & 1.26 & $<0.0001$ \\
\hline West & 1.26 & 1.24 & 1.29 & $<0.0001$ \\
\hline \multicolumn{5}{|c|}{ Comorbidities of Patients } \\
\hline Diabetes Mellites & 0.49 & 0.49 & 0.50 & $<0.0001$ \\
\hline Hypertension & 1.73 & 1.70 & 1.76 & $<0.0001$ \\
\hline Obesity & 0.83 & 0.81 & 0.84 & $<0.0001$ \\
\hline Drug Abuse/Dependence & 1.03 & 0.99 & 1.07 & 0.1261 \\
\hline Current Alcohol Dependence & 0.91 & 0.88 & 0.94 & $<0.0001$ \\
\hline Past History of Alcohol & 0.83 & 0.70 & 0.98 & 0.0265 \\
\hline Current Smoker & 1.30 & 1.27 & 1.32 & $<0.0001$ \\
\hline Past History of Smoking & 0.73 & 0.72 & 0.75 & $<0.0001$ \\
\hline $\begin{array}{l}\text { Acquired immune deficiency } \\
\text { syndrome }\end{array}$ & 0.12 & 0.10 & 0.13 & $<0.0001$ \\
\hline Renal Failure & 0.27 & 0.27 & 0.28 & $<0.0001$ \\
\hline Atrial Fibrillation & 1.13 & 1.11 & 1.14 & $<0.0001$ \\
\hline Hemorrhagic Stroke & 2.49 & 2.39 & 2.60 & $<0.0001$ \\
\hline History of TIA/Stroke & 1.56 & 1.53 & 1.59 & $<0.0001$ \\
\hline \multicolumn{5}{|c|}{ Deyo's Charlson Comorbidity Index (CCI) } \\
\hline 1 & \multicolumn{4}{|c|}{ Reference } \\
\hline 2 & 1.86 & 1.83 & 1.89 & $<0.0001$ \\
\hline 3 & 4.39 & 4.30 & 4.48 & $<0.0001$ \\
\hline 4 & 7.39 & 7.22 & 7.57 & $<0.0001$ \\
\hline$\geq 5$ & 9.38 & 9.18 & 9.58 & $<0.0001$ \\
\hline Area under the ROC curve/c-index & \multicolumn{4}{|c|}{0.882} \\
\hline
\end{tabular}




\subsection{LDs and Post-AIS Outcomes Amongst AIS Population from Year 2003-2014}

We found a total of 4,224,924 hospitalizations due to AIS from year 2003 to 2014 after excluding patients with age $<18$ years and admissions with missing data for age, gender, and race (Figure 1B). Out of 4,224,924 AIS hospitalizations, 451,645 (10.69\%) had LDs.

We analyzed prevalence trends of LDs in AIS hospitalizations. As shown in Figure 2, trends of LDs in AIS hospitalizations were slightly declining from years 2003 to 2014 . LDs prevalence percentage declined from $12.17 \%$ in 2003 to $9.31 \%$ in 2014 ( $p$-Trend $<0.0001)$.

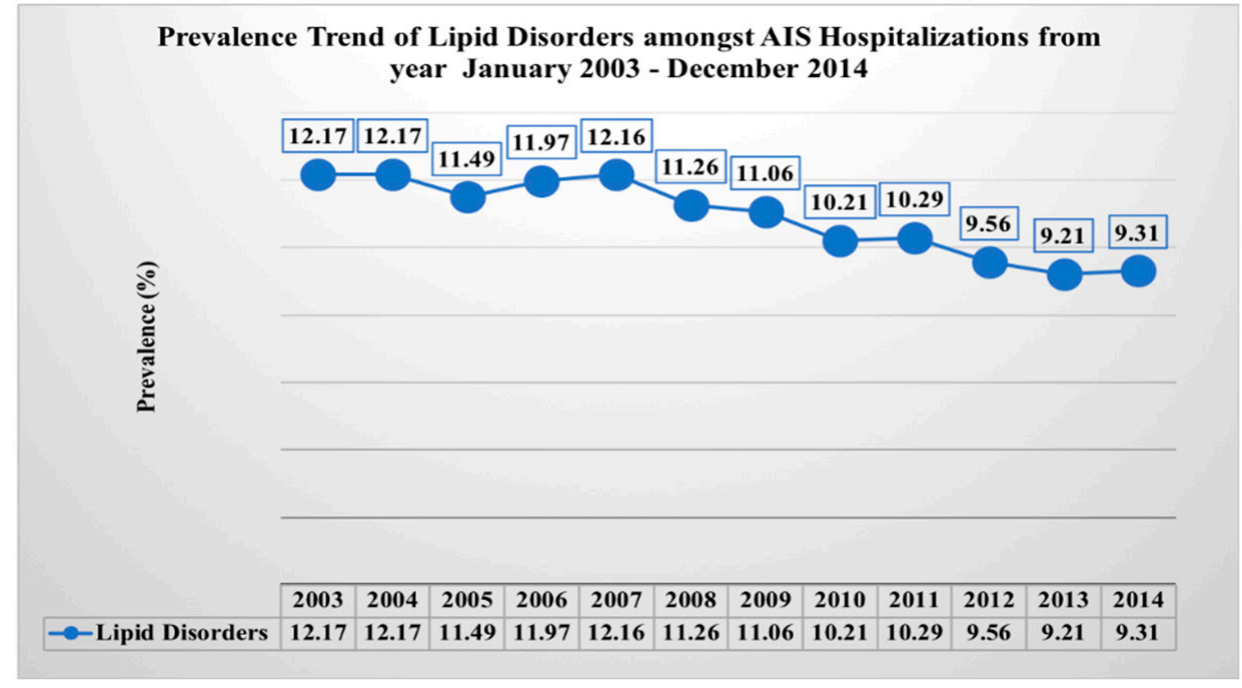

Figure 2. Prevalence trend of lipid disorders.

AIS hospitalizations with LDs were more likely to be female $(49.35 \%$ vs. $46.85 \%, p<0.0001)$. There was no difference in other demographic characteristics like age and race among AIS hospitalizations with and without hyperlipidemia. Co-morbidities such as diabetes $(40.02 \%$ vs. 33.50\%, $p<0.0001)$, hypertension $(86.44 \%$ vs. $78.66 \%, p<0.0001)$, and obesity $(9.85 \%$ vs. $7.63 \%, p<0.0001)$ were higher in patients with LDs than those without LDs. AIS hospitalizations in large, urban (teaching and non-teaching) hospitals and in the Northeast region were more likely to have patients with LDs (Table 3).

Table 3. Characteristics of patients with lipid disorders (LDs) in AIS population from January 2003December 2014.

\begin{tabular}{ccccc}
\hline & LDs & Non-LDs & Total & $p$ Value \\
\hline AIS (\%) & $451,645(10.69)$ & $3,773,279(89.31)$ & $446,446(100)$ & $<0.0001$ \\
\hline \multicolumn{7}{c}{ Demographics of Patients } & & $<0.0001$ \\
\hline Mean Age \pm Standard Error (Years) & $70 \pm 0.04$ & $71 \pm 0.01$ & $<0.0001$ \\
\hline Gender (\%) & & & \\
\hline Female & $222,900(49.35)$ & $1,767,703(46.85)$ & $1,990,602(47.12)$ & \\
Male & $228,746(50.65)$ & $2,005,507(53.15)$ & $2,234,253(52.88)$ & \\
\hline Race (\%) & & & \\
White & $318,230(72.36)$ & $2,667,898(72.54)$ & $2,986,128(72.53)$ & \\
African American & $70,736(16.08)$ & $620,354(16.87)$ & $691,090(16.78)$ & \\
Hispanic & $35,048(7.97)$ & $276,724(7.52)$ & $311,772(7.57)$ & \\
Asian or Pacific Islander & $13,944(3.17)$ & $94,600(2.57)$ & $108,544(2.64)$ & \\
Native American & $1832(0.42)$ & $18,001(0.49)$ & $19,833(0.48)$ &
\end{tabular}


Table 3. Cont

\begin{tabular}{|c|c|c|c|c|}
\hline & LDs & Non-LDs & Total & $p$ Value \\
\hline \multicolumn{5}{|c|}{ Characteristics of Patients } \\
\hline $\begin{array}{l}\text { Median Household Income Category for } \\
\text { patient's Zip code }(\%)\end{array}$ & & & & $<0.0001$ \\
\hline 0-25th percentile & $116,887(26.40)$ & $1,125,147(30.48)$ & $1,242,034(30.04)$ & \\
\hline 26-50th percentile & $105,237(23.77)$ & $959,992(26)$ & $1,065,230(25.76)$ & \\
\hline $51-75$ th percentile & $108,373(24.48)$ & $855,077(23.16)$ & $963,450(23.30)$ & \\
\hline 76-100th percentile & $112,216(25.35)$ & $751,704(20.36)$ & $863,920(20.89)$ & \\
\hline Primary Payer (\%) & & & & $<0.0001$ \\
\hline Medicare & $290,927(64.49)$ & $2,532,866(67.25)$ & $2,823,793(66.95)$ & \\
\hline Medicaid & $28,514(6.32)$ & $257,590(6.84)$ & $286,104(6.78)$ & \\
\hline Private Insurance & $101,488(22.50)$ & $697,741(18.53)$ & $799,229(18.95)$ & \\
\hline Other/Self-pay/No charge & $30,179(6.69)$ & $278,274(7.39)$ & $308,453(7.31)$ & \\
\hline Admission type (\%) & & & & 0.0002 \\
\hline Non-elective & $433,571(96.20)$ & $3,589,986(95.35)$ & $4,023,557(95.44)$ & \\
\hline Elective & $17,130(3.80)$ & $175,254(4.65)$ & $192,384(4.56)$ & \\
\hline Admission day (\%) & & & & 0.0026 \\
\hline Weekday & $337,044(74.63)$ & $2,808,044(74.42)$ & $3,145,089(74.44)$ & \\
\hline Weekend & $114,601(25.37)$ & $965,234(25.58)$ & $1,079,835(25.56)$ & \\
\hline \multicolumn{5}{|c|}{ Characteristics of Hospitals } \\
\hline Bed-size of hospital (\%) ${ }^{\dagger}$ & & & & $<0.0001$ \\
\hline Small & $50,403(11.19)$ & $448,170(11.93)$ & $498,573(11.85)$ & \\
\hline Medium & $115,506(25.63)$ & $963,139(25.64)$ & $1,078,644(25.64)$ & \\
\hline Large & $284,703(63.18)$ & $2,345,109(62.43)$ & $2,629,813(62.51)$ & \\
\hline Hospital Location \& Teaching Status (\%) & & & & $<0.0001$ \\
\hline Rural & $41,205(9.14)$ & $453,884(12.08)$ & 495,089 (11.77) & \\
\hline Urban Non-teaching & $200,443(44.48)$ & $1,582,234(42.12)$ & $1,782,676(42.37)$ & \\
\hline Urban Teaching & $208,964(46.37)$ & $1,720,301(45.80)$ & $1,929,265(45.86)$ & \\
\hline Hospital Region (\%) & & & & $<0.0001$ \\
\hline Northeast & $117,433(26)$ & $778,923(20.64)$ & $896,356(21.22)$ & \\
\hline Midwest & $74,569(16.51)$ & $655,617(17.38)$ & $730,186(17.28)$ & \\
\hline South & $179,036(39.64)$ & $1,631,363(43.23)$ & $1,810,399(42.85)$ & \\
\hline West & $80,608(17.85)$ & $707,375(18.75)$ & $787,983(18.65)$ & \\
\hline Comorbidities of Patients (\%) & & & & $<0.0001$ \\
\hline Diabetes & $179,812(40.02)$ & $1,258,315(33.50)$ & $1,438,128(34.20)$ & \\
\hline Drug abuse & $6735(1.50)$ & $82,624(2.20)$ & $89,359(2.12)$ & \\
\hline Obesity & $44,246(9.85)$ & $286,635(7.63)$ & $330,881(7.81)$ & \\
\hline Hypertension & $388,411(86.44)$ & $2,954,771(78.66)$ & $3,343,182(79.50)$ & \\
\hline Renal failure & $45,347(10.09)$ & $445,605(11.86)$ & $490,952(11.67)$ & \\
\hline Acquired immune deficiency syndrome & $457(0.10)$ & $7606(0.20)$ & $8063(0.19)$ & \\
\hline Deyo's Charlson Comorbidity Index (CCI) & & & & $<0.0001$ \\
\hline 1 & $126,878(28.09)$ & $1,059,432(28.08)$ & $1,186,309(28.08)$ & \\
\hline 2 & $110,148(24.39)$ & $831,212(22.03)$ & $941,360(22.38)$ & \\
\hline 3 & $93,546(20.71)$ & $816,642(21.64)$ & $910,188(21.54)$ & \\
\hline 4 & $65,014(14.39)$ & $537,270(14.24)$ & $602,284(14.26)$ & \\
\hline$\geq 5$ & $56,061(12.41)$ & $528,722(14.01)$ & $584,783(13.84)$ & \\
\hline
\end{tabular}

* This represents a quartile classification of the estimated median household income of residents in the patient's ZIP code; + Bed-size of hospital indicates number of hospital beds which varies depending on hospital location (rural/urban), teaching status (teaching/non-teaching) and region (Northeast/Midwest/Southern/Western). The percentage in brackets are column \% indicating direct comparison between LDs vs. non-LDs amongst AIS patients.

Table 4 includes outcomes of LDs among AIS hospitalizations. Our outcomes of interest were to identify post-AIS outcomes (in-hospital mortality, discharge, disability, risk of death) and complications (post stroke early epilepsy, stroke associated pneumonia, hemorrhagic transformation and upper 
gastro-intestinal bleeding) amongst AIS hospitalizations. All cause in-hospital mortality was lower in AIS with LDs $(2.93 \%$ vs. $5.48 \%, p<0.0001)$ than without LDs. Some $43.14 \%$ of patients with LDs had discharged to home compared to $36.81 \%$ without LDs $(p<0.0001)$. Overall, AIS hospitalizations with LDs had a higher prevalence of discharge to home or routine $(43.14 \% \mathrm{vs} .36 .81 \%, p<0.0001)$ and a lower prevalence of discharge other than home $(56.86 \%$ vs. $63.19 \%, p<0.0001)$ compared to patients without LDs. The prevalence of major/severe loss of function was lower (29.92\% vs. 37.75\%, $p<0.0001)$ among AIS hospitalizations with LDs than without LDs. The patients with LDs was also associated with lower prevalence major/extreme likelihood of death $(15.8 \%$ vs. $21.89 \%, p<0.0001)$ in AIS hospitalizations. Prevalence of post stroke early epilepsy (4.68\% vs. $6.13 \%, p<0.0001)$, SAP $(2.14 \%$ vs. $3.70 \%, p<0.0001)$, HT $(1.25 \%$ vs. $1.71 \%, p<0.0001)$ and UGIB $(0.33 \%$ vs. $0.45 \%, p<0.0001)$ were lower among AIS hospitalizations with LDs than without LDs.

Table 4. Univariate analysis of outcomes of patients with lipid disorders (LDs) among AIS admissions from January 2003-December 2014.

\begin{tabular}{|c|c|c|c|c|}
\hline & LDs & No-LDs & Total & $p$ Value \\
\hline \multicolumn{5}{|c|}{ Post-AIS Outcomes } \\
\hline All Cause in Hospital Mortality (\%) & $13,218(2.93)$ & $206,346(5.48)$ & $219,564(5.21)$ & $<0.0001$ \\
\hline Discharge Disposition (\%) & & & & $<0.0001$ \\
\hline Routine/Home & $187,568(43.14)$ & $1,299,013(36.81)$ & $1,486,581(37.50)$ & \\
\hline Transfer to Short-term Hospital & $12,474(2.87)$ & $114,476(3.24)$ & $126,950(3.20)$ & \\
\hline Transfer to SNF/ICF/Another Type of Facility & $175,573(40.38)$ & $1,639,408(46.45)$ & $1,814,981(45.79)$ & \\
\hline Home Health Care & $59,159(13.61)$ & $476,296(13.50)$ & $535,455(13.51)$ & \\
\hline Discharge other than Home (\%) & $247,206(56.86)$ & $2,230,180(63.19)$ & $2,477,386(62.50)$ & $<0.0001$ \\
\hline APR-DRG Severity/Loss of Function (\%) & & & & $<0.0001$ \\
\hline Minor loss of function & $58,647(13.47)$ & $401,109(11.32)$ & $459,756(11.55)$ & \\
\hline Moderate loss of function & $246,559(56.61)$ & $1,805,199(50.93)$ & $2,051,758(51.55)$ & \\
\hline Major loss of function & $114,404(26.27)$ & $1,106,595(31.22)$ & $1,220,999(30.68)$ & \\
\hline Severe loss of function & $15,899(3.65)$ & $231,623(6.53)$ & $247,522(6.22)$ & \\
\hline Major/Severe Loss of Function/Severity (\%) & $130,303(29.92)$ & $1,338,218(37.75)$ & $1,468,521(36.9)$ & \\
\hline APR-DRG Likelihood of Death (\%) & & & & $<0.0001$ \\
\hline Minor likelihood of death & $171,426(39.36)$ & $1,124,229(31.72)$ & $1,295,655(32.55)$ & \\
\hline Moderate likelihood of death & $195,248(44.83)$ & $1,644,501(46.40)$ & $1,839,749(46.22)$ & \\
\hline Major likelihood of death & $54,708(12.56)$ & $579,523(16.35)$ & $634,231(15.94)$ & \\
\hline Severe likelihood of death & $14,128(3.24)$ & $196,272(5.54)$ & $210,401(5.29)$ & \\
\hline Major/Extreme likelihood of death (\%) & $68,836(15.8)$ & $775,795(21.89)$ & $844,632(21.23)$ & \\
\hline \multicolumn{5}{|c|}{ Post-AIS Complications } \\
\hline Post-stroke early epilepsy & $21,149(4.68)$ & $231,487(6.13)$ & $252,636(5.98)$ & $<0.0001$ \\
\hline Stroke associated pneumonia & $9616(2.13)$ & $139,553(3.70)$ & $149,169(3.53)$ & $<0.0001$ \\
\hline Hemorrhagic Transformation & $5642(1.25)$ & $64,576(1.71)$ & $70,218(1.66)$ & $<0.0001$ \\
\hline Upper gastro-intestinal bleeding & $1477(0.33)$ & $17,152(0.45)$ & $18,629(0.44)$ & $<0.0001$ \\
\hline Length of Stay \pm SE (Days) & $4.83 \pm 0.02$ & $5.43 \pm 0.01$ & & $<0.0001$ \\
\hline Cost of Hospitalization \pm SE (\$) & $34,604 \pm 154$ & $38,547 \pm 62.04$ & & $<0.0001$ \\
\hline
\end{tabular}

APR-DRG: All Patient Refined Diagnosis Related Groups; SNF: Skilled nursing facility; ICF: Intermediate care facility; SE: standard error. The percentage in brackets are column \% indicating direct comparison between LDs vs. non-LDs amongst AIS patients.

Mean length of stay (4.83 days vs. 5.83 days, $p<0.0001)$ and total cost of hospitalization were lower amongst patients with LDs ( $\$ 34,604$ vs. 38,547, $p<0.0001)$ (Table 4). 
Table 5 lists multivariate analysis of outcomes and complications in AIS hospitalizations. LDs were associated with lower adjusted odds of all cause in-hospital mortality (aOR: 0.66, 95\% CI: 0.62-0.69, $p<0.0001$ ), discharge disposition (home vs. no-home) (aOR: 0.83, 95\% CI: 0.82-0.85, $p<0.0001$ ), APR-DRG loss of function (major/severe vs. minor/moderate) (aOR: 0.80, 95\% CI: 0.79-0.82, $p<0.0001$ ), and APR-DRG risk of death (major/severe likelihood vs. minor/moderate likelihood) (aOR: 0.77, 95\% CI: $0.75-0.79, p<0.0001)$ in comparison to patients without LDs amongst AIS hospitalizations of year 2003-2014 (Models 1-4).

Table 5. Multivariate logistic regression analysis of outcomes and complications in patients with LDs compared to non-LDs (reference) amongst AIS hospitalizations.

\begin{tabular}{|c|c|c|c|c|}
\hline \multirow[t]{2}{*}{ Odds Ratio } & \multicolumn{2}{|c|}{ 95\% Confidence Interval } & \multirow[t]{2}{*}{$p$ Value } & \multirow[t]{2}{*}{ Area under the ROC Curve/c-Index } \\
\hline & Lower Limit & Upper Limit & & \\
\hline \multicolumn{5}{|c|}{ Model 1: All cause in-hospital Mortality } \\
\hline 0.66 & 0.62 & 0.69 & $<0.0001$ & 0.76 \\
\hline \multicolumn{5}{|c|}{ Model 2: Discharge Disposition (Home vs. no-Home) } \\
\hline 0.83 & 0.82 & 0.85 & $<0.0001$ & 0.76 \\
\hline \multicolumn{5}{|c|}{ Model 3: APR-DRG loss of function (major/severe vs. minor/moderate) } \\
\hline 0.80 & 0.79 & 0.82 & $<0.0001$ & 0.82 \\
\hline \multicolumn{5}{|c|}{ Model 4: APR-DRG risk of death (major/severe likelihood vs. minor/moderate likelihood) } \\
\hline 0.77 & 0.75 & 0.79 & $<0.0001$ & 0.81 \\
\hline \multicolumn{5}{|c|}{ Model 5: Post Stroke Early Epilepsy } \\
\hline 0.89 & 0.8 & 0.86 & $<0.0001$ & 0.65 \\
\hline \multicolumn{5}{|c|}{ Model 6: Stroke Associated Pneumonia } \\
\hline 0.75 & 0.71 & 0.80 & $<0.0001$ & 0.8 \\
\hline \multicolumn{5}{|c|}{ Model 7: Upper GI Bleeding } \\
\hline 0.85 & 0.73 & 0.99 & $<0.0001$ & 0.69 \\
\hline \multicolumn{5}{|c|}{ Model 8: Hemorrhagic Transformation } \\
\hline 0.82 & 0.75 & 0.89 & $<0.0001$ & 0.78 \\
\hline
\end{tabular}

All models are adjusted for demographics (age, gender, race), patient-level hospitalization variables (admission day, primary payer, admission type, median household income category), hospital-level variables (hospital region, teaching versus non-teaching hospital, hospital bed-size), comorbidities, concurrent conditions like hypertension, diabetes mellitus, hypercholesterolemia, atrial fibrillation, obesity, amyloidosis, hemorrhagic transformation, smoking status, drug abuse, alcohol abuse, medication use (anticoagulant and antiplatelet medication, platelets inhibitor infusion, chronic use of aspirin), and use of IV tPA during the same hospitalization or in a different institution within the $24 \mathrm{~h}$ prior to admission to the facility, mechanical thrombectomy, gastrostomy, nasogastric tube insertion, invasive-noninvasive mechanical ventilation, and Charlson's co-morbidity index (CCI).

LDs were associated with lower adjusted odds of post stroke early epilepsy (aOR:0.89, 95\% CI: 0.8-0.86, $p<0.0001$ ), SAP (aOR: 0.75, 95\% CI: 0.71-0.80, $p<0.0001)$, upper GI bleeding (aOR:0.85, 95\% CI: $0.73-0.99, p<0.0001$ ) and hemorrhagic transformation (aOR: 0.82, 95\% CI: 0.75-0.89, $p<0.0001$ ) in comparison to patients without LDs (Models 5-8).

C statistic was used to validate the accuracy of the regressions. All models have c-index $>0.6$, which indicates a good model fit (Table 5).

\section{Discussion}

In this study, we aimed to investigate the link between acute ischemic stroke (AIS) and lipid disorders (LDs), specifically the odds of having AIS with LDs, and whether LDs were associated with better outcomes or less complications in AIS patients as compared to those without LDs. We 
did this by performing a population-based retrospective cross-sectional analysis of the NIS in adult hospitalizations for AIS.

Our study found that those with LDs had a higher prevalence of being hospitalized with AIS as compared to those without LDs. Co-morbidities, such as diabetes, hypertension, obesity, and depression were also higher in those with LDs than in those without them. Our findings are similar to a study by Olsen et al. who have reported that patients with hypercholesterolemia are at increased risk of stroke [22]. However, the paradoxical relationship is evident when analyzing for post-AIS outcomes such as in-hospital mortality, discharge status, disability, and risk of death, as well as post-AIS complications such as post-stroke early epilepsy, stroke associated pneumonia, hemorrhagic transformation, and upper GI bleeding.

For AIS patients with lipid disorders, there was reduced in-hospital mortality (aOR: 0.66, $p<0.0001$ ), increased discharge to home (aOR: $0.83, p<0.0001$ ), decreased major/severe loss of function (aOR: 0.80, $p<0.0001$ ), and decreased major/extreme likelihood of death (aOR: 0.77, $p<0.0001$ ); additionally, there was reduced risk of post stroke epilepsy (aOR:0.89, $p<0.0001$ ), SAP (aOR: 0.75, $p<0.0001)$, upper GI bleeding (aOR:0.85, $p<0.0001$ ) and hemorrhagic transformation (aOR: 0.82, $p<0.0001)$ as compared to AIS patients without LDs. There are supporting studies to our paradoxical findings that have reported higher cholesterol levels on admission are associated with better long-term survival or outcomes amongst AIS survivors [22,28,29].

There is thus an incongruity that is seen in AIS patients; on one hand, there is a benefit to reducing cholesterol and LDs in that there is a reduced prevalence of AIS and co-morbidities as compared to those without LDs, as well as for being beneficial for cerebrovascular mortality and morbidity $[3,4]$. However, patients with LDs have better post-AIS outcomes and less post-AIS complications.

This paradox may be explained by the type of stroke that is often seen with those with LDs. Those with LDs may be more predisposed toward small-vessel strokes, and thus will have less severe strokes with better prognoses; as a result, they would have improved post-AIS outcomes and reduced post-AIS complications as compared to those without LDs who may be more prone to the often more severe large brain vessel occlusion. This theory can be supported by our data, as well as that large occlusion of brain vessels as seen in cardio embolic strokes have the lowest total serum cholesterol levels [23,24].

Interestingly, the trends of LDs in AIS hospitalizations have decreased from 2003-2014. This may be due to reduced hospitalizations for minor strokes and may support theory that those with LDs are more prone to small-vessel strokes which have better prognoses. Thus, those patients with LDs and minor strokes are often not being hospitalized as much, and if they are, they will show improved post-AIS outcomes and reduced complications as shown in our study. However, in our study AIS was analyzed as a whole, and was not classified into subtypes using TOAST classification. This was one limitation of our study to support the decreasing trends and theory of LDs association with small vessel stroke which have better prognoses.

This analysis and confirmation of the paradoxical relationship seen between LDs and AIS may have implications for treatment strategies in the future. Statins are certainly beneficial to cardiovascular health, have been proven to have neuroprotective and microvascular benefits in animal stroke models, and may be important in augmenting cerebral repair after ischemic injury [20]. They also have been proven to improve survival for up to a year after stroke.

Further work must be done in order to fully determine why LDs seem to have a beneficial effect on AIS outcomes and complications, and to determine potential treatment strategies. Clinical trials should be run to determine the effects of LDs and statins on AIS, AIS subtypes such as small-vessel disease, and AIS outcomes and complications.

A major strength of the study was that findings were nationally representative for the USA. NIS data is a large deidentified inpatient database, and our study has good statistical power. The APR-DRG coding systems used in this study to assess the severity of illness and risk of mortality are externally validated. They are very reliable and consistent, and widely used by hospitals, consumers, payers and regulators $[30,31]$. 
One main limitation to this study mentioned above, AIS was not classified into subtypes using TOAST classification (such as cardio-embolic, small-vessel, or large artery atherothrombotic strokes). Further investigations should classify AIS into these subtypes and determine if there is an association between LDs and these subtypes. This would be able to determine if theory that LDs cause the less severe small-vessel strokes is correct, and thus is the reason why we see better AIS-outcomes and reduced complications with LDs. Another limitation of the study was LDs were identified as a secondary diagnosis (history of LDs or first-time diagnosis) responsible for AIS and we did not have data on statin use prior or during the hospitalization. So neuroprotective role of statin [20] was difficult to differentiate. Other limitations to this study include the fact that we only considered patients who were hospitalized with strokes; between $10 \%-40 \%$ of stroke patients are not admitted to the in-patient hospital [13]. Thus, patients with less severe strokes may not be included in this study. The outcomes evaluated while patients were in hospital and status of patients on discharge, we had not evaluated the long-term outcomes. Additionally, although co-morbidities were accounted for and adjusted for in analysis, other subtler differences between patients such as prior strokes or other unaccounted medications may flaw our design outcomes. Though we had adjusted outcomes model with antiplatelets or anticoagulant use before and during hospitalization, but we had no record on statin use to adjust the models.

\section{Conclusions}

LDs have been shown to have an increased prevalence in patients with AIS hospitalizations as compared to those without LDs. However, we have also confirmed that LDs have paradoxically been shown to improve post-AIS outcomes and reduce post-AIS complications. This complicated relationship between stroke and LDs requires further work and research to determine the reasoning as to the associated benefit between higher lipids and better outcomes, and whether the theory in that LDs have a stronger association in causing small vessel and thus less severe strokes with better prognoses can be confirmed. Clinical trials should be undertaken to further determine the relationship of statin uses and outcomes to refine treatment strategies before and after stroke onset, and further work should be initiated to determine the association between LDs and AIS subtypes such as small-vessel strokes, AIS-subtype outcomes, and AIS-subtype complications.

Supplementary Materials: The following are available online at http://www.mdpi.com/1010-660X/55/8/475/s1. Table S1: ICD-9-CM codes used in this analysis, Table S2: Deyo's modification of Charlson's co-morbidity index (CCI).

Author Contributions: For conceptualization, U.P.; methodology, U.P., M.S.D. (Mandip S. Dhamoon); software, U.P., P.M.; validation, M.D.; formal analysis, U.P.; investigation, U.P., P.M.; resources, M.S.D. (Mandip S. Dhamoon); data curation, U.P., M.D.; writing—original draft preparation, U.P., M.S.D. (Matthew S. DeMasi), M.D.; writing-review and editing, A.L., V.B.J., M.S.D. (Mandip S. Dhamoon); visualization, U.P., P.M.; supervision, A.L., V.B.J.; project administration, P.M.; funding acquisition, none.

Funding: This research received no external or internal funding.

Conflicts of Interest: The authors declare no conflict of interest.

\section{References}

1. Benjamin, E.J.; Blaha, M.J.; Chiuve, S.E.; Cushman, M.; Das, S.R.; Deo, R.; de Ferranti, S.D.; Floyd, J.; Fornage, M.; Gillespie, C.; et al. Heart Disease and Stroke Statistics-2017 Update: A Report From the American Heart Association. Circulation 2017, 135, e146-e603. [CrossRef] [PubMed]

2. Yang, Q.; Tong, X.; Schieb, L.; Vaughan, A.; Gillespie, C.; Wiltz, J.L.; King, S.C.; Odom, E.; Merritt, R.; Hong, Y.; et al. Vital Signs: Recent Trends in Stroke Death Rates-United States, 2000-2015. MMWR Morb. Mortal. Wkly. Rep. 2017, 66, 933-939. [CrossRef] [PubMed]

3. Klag, M.J.; Ford, D.E.; Mead, L.A.; He, J.; Whelton, P.K.; Liang, K.Y.; Levine, D.M. Serum cholesterol in young men and subsequent cardiovascular disease. N. Engl. J. Med. 1993, 328, 313-318. [CrossRef] [PubMed]

4. Castelli, W.P.; Anderson, K.; Wilson, P.W.; Levy, D. Lipids and risk of coronary heart disease. The Framingham Study. Ann. Epidemiol. 1992, 2, 23-28. [CrossRef] 
5. Hankey, G.J.; Warlow, C.P. Treatment and secondary prevention of stroke: Evidence, costs, and effects on individuals and populations. Lancet 1999, 354, 1457-1463. [CrossRef]

6. Prospective Studies Collaboration. Cholesterol, diastolic blood pressure, and stroke: 13,000 strokes in 450,000 people in 45 prospective cohorts. Lancet 1995, 346, 1647-1653. [CrossRef]

7. Landau, W.M. Is cholesterol a risk factor for stroke?: No. Arch. Neurol. 1999, 56, 1521-1524. [CrossRef] [PubMed]

8. Amarenco, P. Hypercholesterolemia, lipid-lowering agents, and the risk for brain infarction. Neurology 2001, 57, S35-S44. [CrossRef] [PubMed]

9. Wolf, P.A.; Kannel, W.B.; Verter, J. Current status of risk factors for stroke. Neurol. Clin. 1983, 1, $317-343$. [CrossRef]

10. Kagan, A.; Popper, J.S.; Rhoads, G.G. Factors related to stroke incidence in Hawaii Japanese men. The Honolulu Heart Study. Stroke 1980, 11, 14-21. [CrossRef]

11. Iso, H.; Jacobs, D.R., Jr.; Wentworth, D.; Neaton, J.D.; Cohen, J.D. Serum cholesterol levels and six-year mortality from stroke in 350,977 men screened for the multiple risk factor intervention trial. N. Engl. J. Med. 1989, 320, 904-910. [CrossRef] [PubMed]

12. Benfante, R.; Yano, K.; Hwang, L.J.; Curb, J.D.; Kagan, A.; Ross, W. Elevated serum cholesterol is a risk factor for both coronary heart disease and thromboembolic stroke in Hawaiian Japanese men. Implications of shared risk. Stroke 1994, 25, 814-820. [CrossRef] [PubMed]

13. Schulz, U.G.; Rothwell, P.M. Differences in vascular risk factors between etiological subtypes of ischemic stroke: Importance of population-based studies. Stroke 2003, 34, 2050-2059. [CrossRef] [PubMed]

14. Tirschwell, D.L.; Smith, N.L.; Heckbert, S.R.; Lemaitre, R.N.; Longstreth, W.T., Jr.; Psaty, B.M. Association of cholesterol with stroke risk varies in stroke subtypes and patient subgroups. Neurology 2004, 63, 1868-1875. [CrossRef] [PubMed]

15. Amarenco, P.; Labreuche, J.; Elbaz, A.; Touboul, P.J.; Driss, F.; Jaillard, A.; Bruckert, E. Blood lipids in brain infarction subtypes. Cereb. Dis. 2006, 22, 101-108. [CrossRef] [PubMed]

16. Eastern Stroke and Coronary Heart Disease Collaborative Research Group. Blood pressure, cholesterol, and stroke in eastern Asia. Lancet 1998, 352, 1801-1807. [CrossRef]

17. Dyker, A.G.; Weir, C.J.; Lees, K.R. Influence of cholesterol on survival after stroke: Retrospective study. BMJ 1997, 314, 1584-1588. [CrossRef] [PubMed]

18. Vauthey, C.; de Freitas, G.R.; van Melle, G.; Devuyst, G.; Bogousslavsky, J. Better outcome after stroke with higher serum cholesterol levels. Neurology 2000, 54, 1944-1949. [CrossRef]

19. Zuliani, G.; Cherubini, A.; Atti, A.R.; Ble, A.; Vavalle, C.; Di Todaro, F.; Benedetti, C.; Volpato, S.; Marinescu, M.G.; Senin, U.; et al. Low cholesterol levels are associated with short-term mortality in older patients with ischemic stroke. J. Gerontol. Ser. A Biol. Sci. Med. Sci. 2004, 59, 293-297. [CrossRef]

20. Ni Chroinin, D.; Callaly, E.L.; Duggan, J.; Merwick, A.; Hannon, N.; Sheehan, O.; Marnane, M.; Horgan, G.; Williams, E.B.; Harris, D.; et al. Association between acute statin therapy, survival, and improved functional outcome after ischemic stroke: The North Dublin Population Stroke Study. Stroke 2011, 42, 1021-1029. [CrossRef]

21. Tuttolomondo, A.; Di Raimondo, D.; Di Sciacca, R.; Pedone, C.; La Placa, S.; Arnao, V.; Pinto, A.; Licata, G. Effects of clinical and laboratory variables at admission and of in-hospital treatment with cardiovascular drugs on short term prognosis of ischemic stroke. The GIFA study. Nutr. Metab. Cardiovasc. Dis. NMCD 2013, 23, 642-649. [CrossRef] [PubMed]

22. Olsen, T.S.; Christensen, R.H.; Kammersgaard, L.P.; Andersen, K.K. Higher total serum cholesterol levels are associated with less severe strokes and lower all-cause mortality: Ten-year follow-up of ischemic strokes in the Copenhagen Stroke Study. Stroke 2007, 38, 2646-2651. [CrossRef] [PubMed]

23. Jorgensen, H.S.; Nakayama, H.; Reith, J.; Raaschou, H.O.; Olsen, T.S. Acute stroke with atrial fibrillation. The Copenhagen Stroke Study. Stroke 1996, 27, 1765-1769. [CrossRef] [PubMed]

24. Lin, H.J.; Wolf, P.A.; Kelly-Hayes, M.; Beiser, A.S.; Kase, C.S.; Benjamin, E.J.; D’Agostino, R.B. Stroke severity in atrial fibrillation. The Framingham Study. Stroke 1996, 27, 1760-1764. [CrossRef] [PubMed]

25. Volpato, S.; Leveille, S.G.; Corti, M.C.; Harris, T.B.; Guralnik, J.M. The value of serum albumin and high-density lipoprotein cholesterol in defining mortality risk in older persons with low serum cholesterol. J. Am. Geriatr. Soc. 2001, 49, 1142-1147. [CrossRef] [PubMed] 
26. Birman-Deych, E.; Waterman, A.D.; Yan, Y.; Nilasena, D.S.; Radford, M.J.; Gage, B.F. Accuracy of ICD-9-CM codes for identifying cardiovascular and stroke risk factors. Med. Care 2005, 43, 480-485. [CrossRef] [PubMed]

27. Oake, J.; Aref-Eshghi, E.; Godwin, M.; Collins, K.; Aubrey-Bassler, K.; Duke, P.; Mahdavian, M.; Asghari, S. Using Electronic Medical Record to Identify Patients With Dyslipidemia in Primary Care Settings: International Classification of Disease Code Matters From One Region to a National Database. Biomed. Inform. Insights 2017, 9, 1178222616685880. [CrossRef]

28. Markaki, I.; Nilsson, U.; Kostulas, K.; Sjostrand, C. High cholesterol levels are associated with improved long-term survival after acute ischemic stroke. J. Stroke Cerebrovasc. Dis. 2014, 23, e47-e53. [CrossRef] [PubMed]

29. Koton, S.; Molshatzki, N.; Bornstein, N.M.; Tanne, D. Low cholesterol, statins and outcomes in patients with first-ever acute ischemic stroke. Cerebrovasc. Dis. 2012, 34, 213-220. [CrossRef] [PubMed]

30. McCormick, P.J.; Lin, H.M.; Deiner, S.G.; Levin, M.A. Validation of the All Patient Refined Diagnosis Related Group (APR-DRG) Risk of Mortality and Severity of Illness Modifiers as a Measure of Perioperative Risk. J. Med. Syst. 2018, 42, 81. [CrossRef] [PubMed]

31. Baram, D.; Daroowalla, F.; Garcia, R.; Zhang, G.; Chen, J.J.; Healy, E.; Riaz, S.A.; Richman, P. Use of the All Patient Refined-Diagnosis Related Group (APR-DRG) Risk of Mortality Score as a Severity Adjustor in the Medical ICU. Clin. Med. Circ. Respir. Pul. Med. 2008, 2, 19-25. [CrossRef]

(C) 2019 by the authors. Licensee MDPI, Basel, Switzerland. This article is an open access article distributed under the terms and conditions of the Creative Commons Attribution (CC BY) license (http://creativecommons.org/licenses/by/4.0/). 